\title{
Synthesis of Dialkenyl-Substituted Double-Decker Silsesquioxanes as Precursors for Linear Copolymeric Systems
}

\author{
Katarzyna Mituła ${ }^{1} \cdot$ Beata Dudziec $^{1,2} \cdot$ Bogdan Marciniec $^{1,2}$
}

Received: 29 September 2017 / Accepted: 18 November 2017 / Published online: 27 November 2017

(c) The Author(s) 2017. This article is an open access publication

\begin{abstract}
In this paper, we report an efficient synthetic route for dialkenyl-substituted double-decker silsesquioxane derivatives as difunctional reagents for further applications. This study concerns synthesis of allyl, hex-5-enyl- and dec-9-enyl-doubledecker silsesquioxanes obtained via condensation and hydrosilylation process as alternative processes, with high yields. The presence of unsaturated groups allows these compounds to be used as molecular synthons for hybrid silsesquioxane based systems of macromolecular nature.
\end{abstract}

\section{Graphical Abstract}

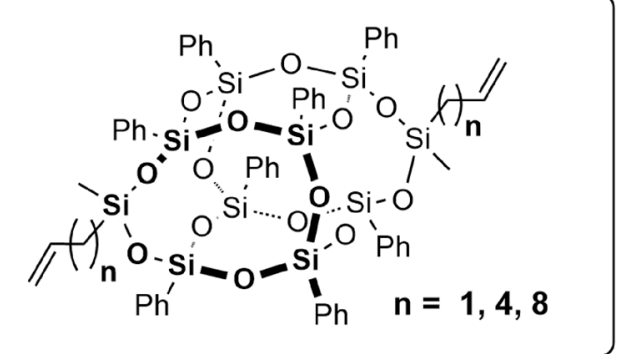

- condensation and hydrosilylation as alternative synthetic routes

- different alkenyl chain length

- high isolated yields

Keywords Silsesquioxane $\cdot$ DDSQ $\cdot$ Double-decker silsesquioxane $\cdot$ Dialkenylfunctional silsesquioxanes

Electronic supplementary material The online version of this article (https://doi.org/10.1007/s10904-017-0746-y) contains supplementary material, which is available to authorized users.

Beata Dudziec

beata.dudziec@gmail.com

Bogdan Marciniec

Bogdan.Marciniec@amu.edu.pl

1 Faculty of Chemistry, Adam Mickiewicz University, Poznan, Umultowska 89B, 61-614 Poznan, Poland

2 Centre for Advanced Technologies, Adam Mickiewicz University, Poznan, Umultowska 89C, 61-614 Poznan, Poland

\section{Introduction}

The compounds of the general formula $\left(\mathrm{RSiO}_{3 / 2}\right)_{\mathrm{n}}$, are known as Polyhedral Oligomeric silsesquioxanes and they constitute a wide class of well-defined inorganic-organic systems. Their nanometric and defined 3D structure of $\mathrm{Si}-\mathrm{O}-\mathrm{Si}$ core and organic coronae make them suitable for classification as hybrid systems. These compounds attract considerable attention among chemists due to their unique properties (physical, chemical and biological) and allow the potential application in many branches of chemistry, biochemistry and mostly in composite materials $[1,2]$. The chemists specialized in of polymers and composites are particularly interested in these compounds as they may serve as polymer modifiers and/or comonomers. Moreover, the resulting materials of a hybrid nature may exhibit attractive and improved physicochemical parameters, i.a. thermal, 
mechanical and optical in comparison with their organic polymer derivatives $[3,4]$. The way of silsesquioxane incorporation into polymer matrix depends on the number and the type of functional groups (FG) attached to the Si-O-Si core [5]. Functional silsesquioxanes may be built into the structure of polymer via chemical bonds either as pendant molecules or cross-linking agents during, e.g. copolymerization reaction (depending on the amount of FG in the silsesquioxane structure), or bonded covalently, so-called "blended into" the matrix when the FG groups are inert. Currently, the most widely described group of silsesquioxanes is the one with closed cage, i.e. completely condensed silsesquioxanes (Fig. 1) of cages form $\mathrm{T}_{6}$ to $\mathrm{T}_{12}$ and $\mathrm{D}_{2} \mathrm{~T}_{8}$-a new type of silsesquioxane called double-decker DDSQ due to its structure $[1,6,7]$.

This difunctional double-decker silsesquioxane has recently attracted considerable interest due to its unique structure and possibility of two functional groups (FG) embodiment of diverse nature and reactivity. This in turns enables application of these silsesquioxanes in synthesis of molecular and macromolecular DDSQ based systems that are characterized with interesting physicochemical properties [8]. The type of reaction employed for modification of DDSQ depends on the kind of functional group attached to the $\mathrm{Si}-\mathrm{O}-\mathrm{Si}$ core and, i.a. $\mathrm{Si}-\mathrm{H}, \mathrm{Si}-\mathrm{HC}=\mathrm{CH}_{2}$ are of special interest as there are reports on efficient catalytic reactions used for synthesis of copolymeric DDSQ based systems (e.g. hydrosilylation, cross-metathesis or silylative coupling) [9-13]. The macromolecular DDSQ-based systems are of specific importance as the silsesquioxane is embedded in the linear chain of the copolymer.
This study was undertaken to design double-decker silsesquioxanes with two reactive alkenyl- substituents and to provide an efficient procedure for their construction. This paper report on synthetic route for synthesis of diallyl-, di(hex5-enyl-) and di(dec-9-enyl-) DDSQ involving condensation and/or hydrosilylation reaction and thorough spectroscopic characterization. These compounds possess specific characteristics allowing their further application as precursors of hybrid matrials, e.g. comonomers for copolymerization reaction with olefins.

\section{Result and Discussion}

Our goal was to synthesize a series of dialkenyl-substituted DDSQ compounds varying in the aspect of the length of the alkenyl group at silsesquioxane core. This modification affects not only differences in the synthetic procedure but also their physical properties. The first stage of our studies was to develop efficient synthetic method for the preparation of alkenylchlorosilanes with longer alkenyl chains, i.e. hex5-enyl- and dec-9-enyl-) which in contrast to allyl analogue, are not commercially available (Scheme 1 ).

Hex-5-enyl- and dec-9-enyl-chlorosilanes we obtained via $\left[\mathrm{Pt}_{2}(\mathrm{dvs})_{3}\right]$ catalyzed hydrosilylation of 1,5 -hexadiene and 1,9-decadiene by $\mathrm{Cl}_{2} \mathrm{SiMeH}$ [14]. The reactions were carried out at room temperature and in the excess of the corresponding diene that served as the reagent and reaction medium (due to ease of isolation procedure). The reaction conditions and catalyst enabled obtaining desired, $\beta$-hydrosilylation product with high selectivity and yields (see Sect. “4”). The

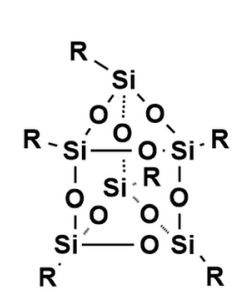

$\mathrm{T}_{6}$

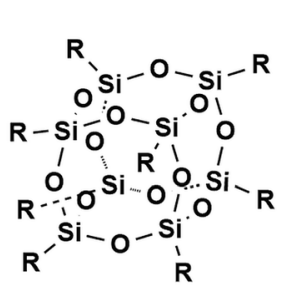

$\mathrm{T}_{8}$
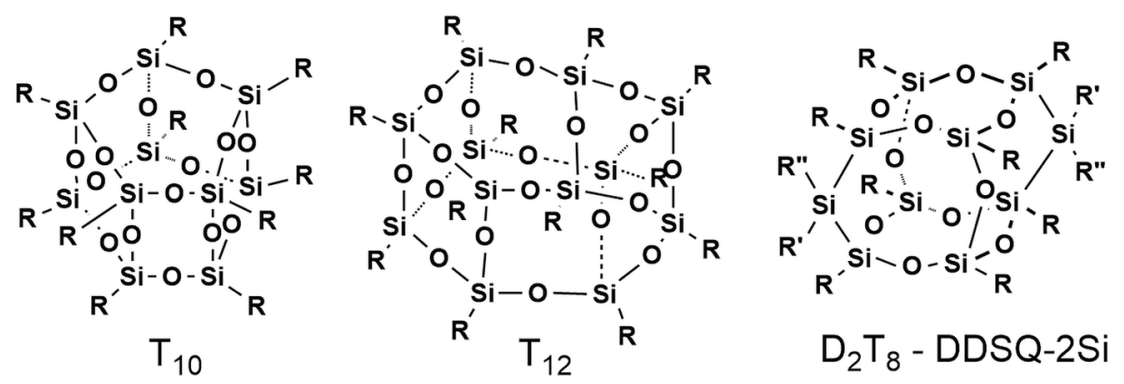

Fig. 1 General structures of completely condensed silsesquioxanes $T_{6}-T_{12}$ and $D_{2} T_{8}$ (DDSQ)

Scheme 1 Synthesis of hex-5enyl- and dec-9-enyl-substituted dichloromethylsilanes

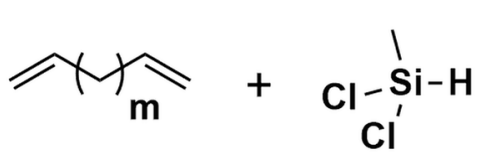

$m=2-1,5-$ hexadiene

$m=6 \quad-1,9-$ decadiene

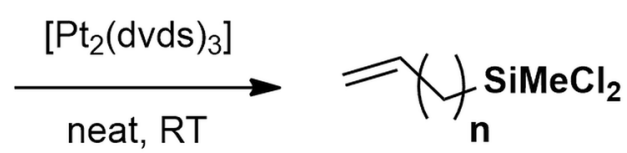

$\mathrm{n}=4$ - hex-5-enylsilane

$\mathrm{n}=8$ - dec-9-enylsilane 
next step was the condensation reaction of dichloroalkenylsilanes with tetrasilanol form of DDSQ (DDSQ-4OH).

Our introductory tests revealed the necessity of using dilute THF solutions of reagents to avoid side reactions. At the initial step of the process the reaction mixture was cooled in an ice bath, the amine and then the chlorosilane was added (Scheme 2). For the effective product formation, the respective chlorosilane was used in 2.1 equiv. per $1 \mathrm{~mol}$ of DDSQ-4OH. Then, the reaction was left to warm up to RT. The condensation progress was verified by FTIR analysis (disappearance of the signals from stretching vibrations of the Si-OH in DDSQ-4OH) (Fig. 2). The crude product was isolated from the reaction mixture by simple filtration and purified by precipitation in methanol.

The condensation procedure was tested using two types of amines, i.e. $\mathrm{N}(i \mathrm{Pr})_{2} \mathrm{Et}$ and $\mathrm{NEt}_{3}$ and the latter was found to be the most efficient. The amount of amine was calculated for 1.5 equiv. per one $\mathrm{Si}-\mathrm{OH}$ moiety.

Interestingly, the DDSQ-2Si-Hexenyl and DDSQ-2SiDecenyl may be also synthesized using an alternative method which allowed to avoid working with chlorosilanes that are susceptible to moisture. We discovered that double-decker silsesquioxane with Si-H (DDSQ-2SiH) reactive bond [11] could be used as a hydrosilylation agent for 1,5-hexadiene and 1,9-decadiene (Scheme 3).

We have tested this option and it proved to be an efficient route in obtaining di(hex-5-enyl-) and di(dec9-enyl-)substituted DDSQ respective compounds with high yields (DDSQ-2Si-Hexenyl-90\% and DDSQ-2SiDecenyl-79\%). To avoid diene hydrosilylation from both sides, a substantial excess of diene (5 equiv. per one $\mathrm{Si}-\mathrm{H}$ moiety) should be applied and this evolves problems with product isolation. The progress of the reaction was monitored using FT-IR analysis (disappearance of the signals from stretching vibrations of the - $\mathrm{Si}-\mathrm{H}$ in DDSQ2SiH) (Fig. 3). The crude product was isolated from the reaction mixture by chromatographic column (silica gel 60) using DCM:hexane 3:2 as an eluent. Evaporation of eluent resulted in an analytically pure product.

The hydrosilylation and condensation reaction were appointed to be alternative and efficient procedures for obtaining desired dialkenylsubstituted DDSQ compounds
Scheme 2 Synthesis of dialkenylfunctional double-decker silsesquioxanes via condensation reaction
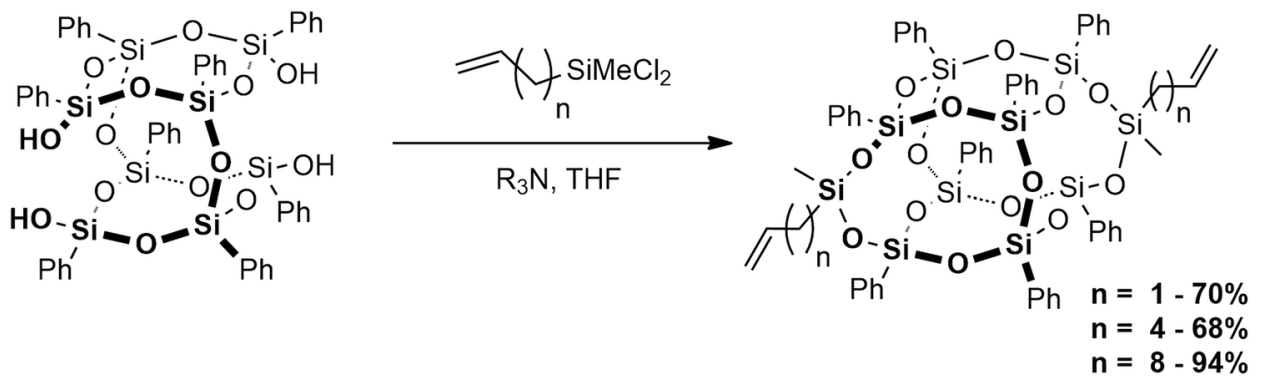

Fig. 2 FT-IR spectra of DDSQ4OH, DDSQ-2Si-Allyl and DDSQ-2Si-Decenyl after completion of condensation reaction

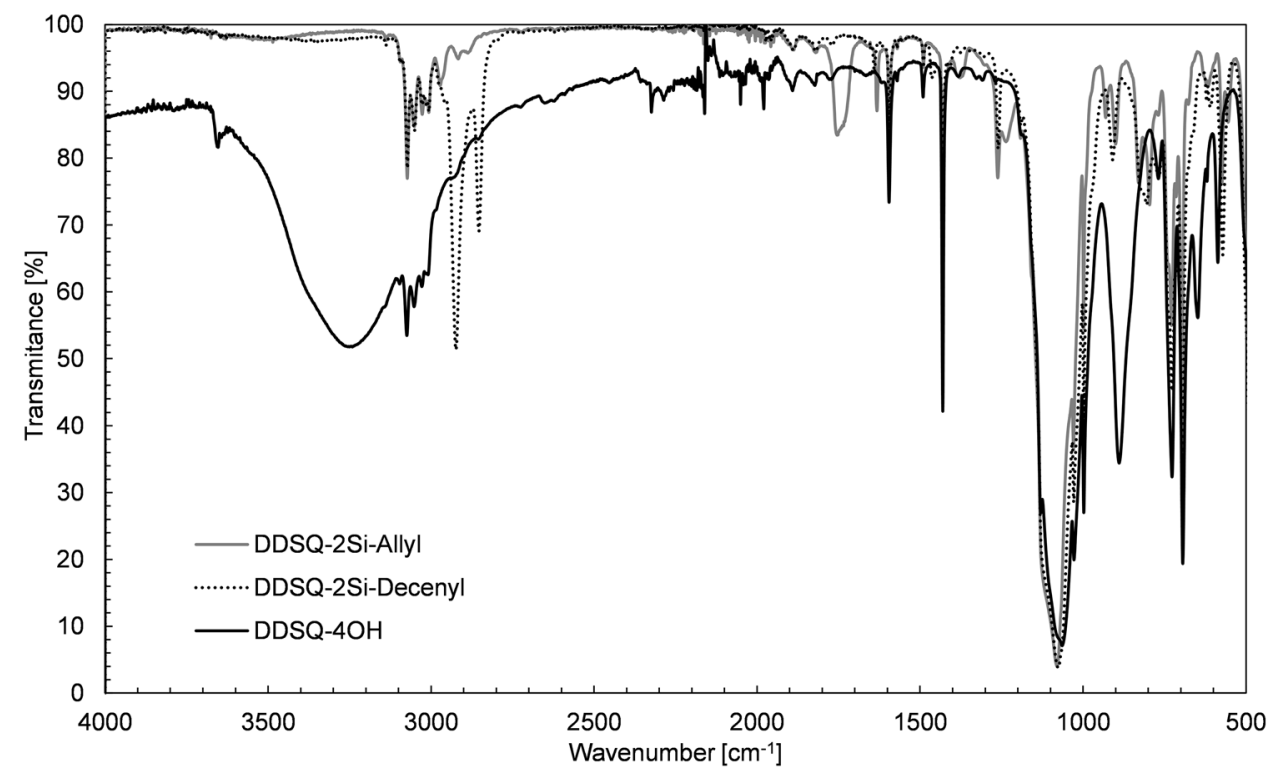




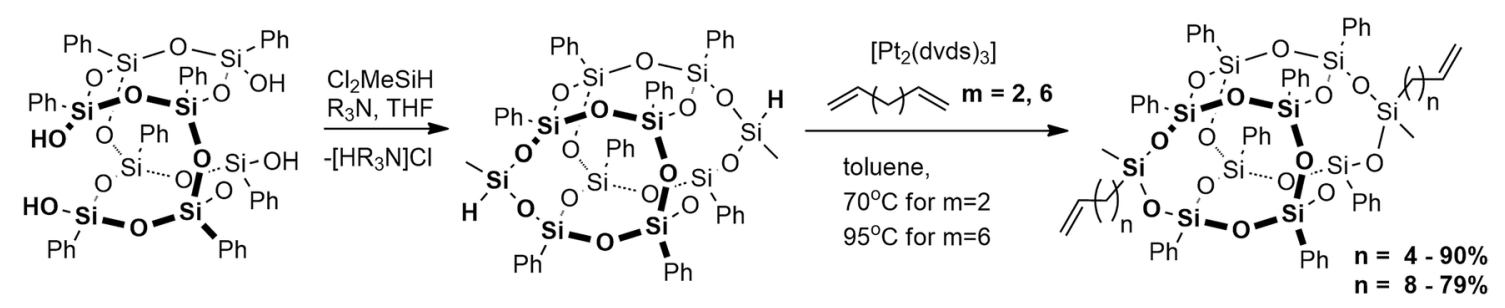

Scheme 3 Synthesis of DDSQ-2Si-Hexenyl and DDSQ-2Si-Decenyl derivatives via hydrosilylation reaction of dienes with DDSQ-2Si-H

Fig. 3 FT-IR spectra of DDSQ-2Si-H and DDSQ-2SiHexenyl after completion of hydrosilylation reaction

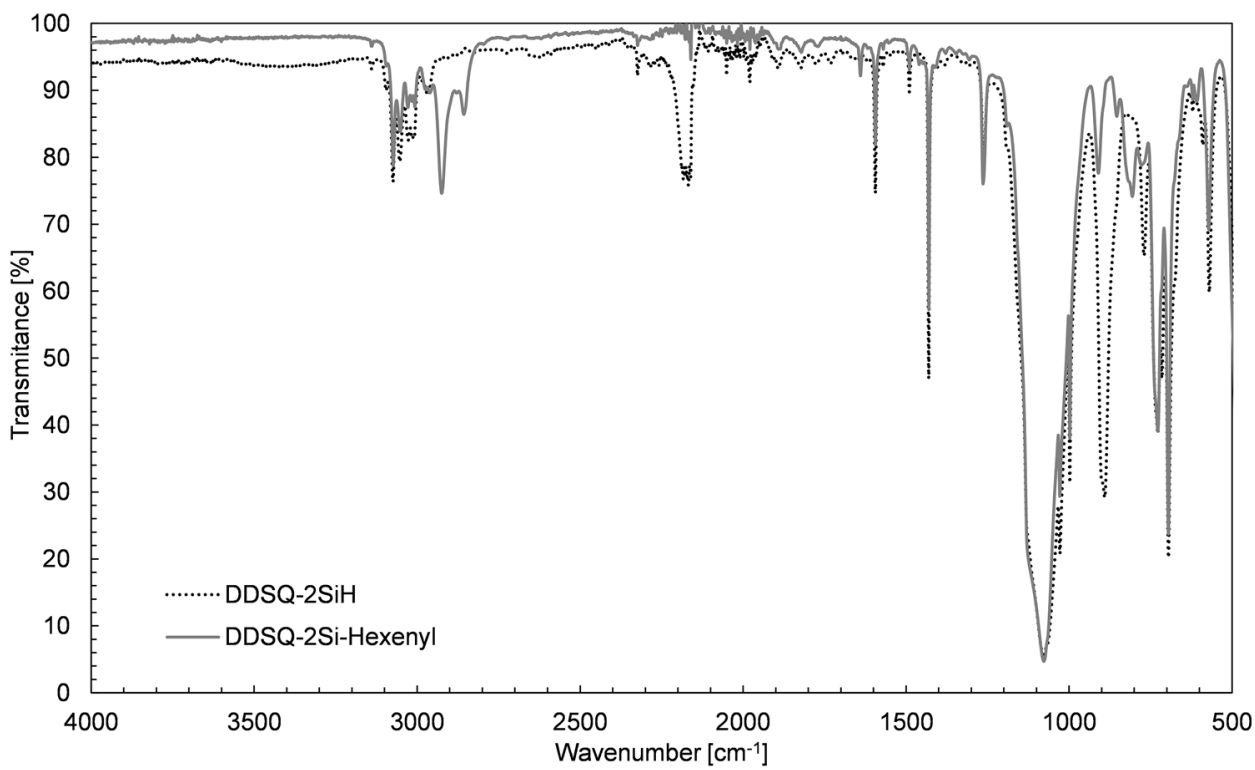

with different alkenyl chain lengths and good and very good isolated yields. Both methods require additional reaction step, i.e. for condensation, the synthesis of respective alkenyldichlorosilane is necessary and for hydrosilylation, the synthesis of DDSQ-2SiH as the relevant reagent is crucial. As DDSQ-4OH is more valuable reagent (small loss of DDSQ-2SiH during isolation procedure) for us, we prefer to apply the condensation reaction for the formation of respective final products. However, it should be noted that condensation reaction is preferred either for chlorosilanes with short alkenyl chain (due to their commercial availability) or for rather longer chain length, as for dichloro(dec-9-enyl)methylsilane. The synthesis of DDSQ-2Si-Hexenyl via condensation reaction with dichloro(hex-5-enyl) methylsilane proceeds with lower efficiency due to the presence of $\alpha$-hydrosilylation product of the resulting chlorosilane. The final DDSQ-2SiHexenyl contains up to $10 \%$ of a product that possesses a 1-methyl-hex-4-enyl- group as one of a substituent. It is visible on the ${ }^{1} \mathrm{H}$ and ${ }^{29} \mathrm{Si}$ NMR spectra (see Supporting Information). The final product could be purified using column chromatography that unfortunately affects the final reaction yield. On the contrary, hydrosilylation of 1,9-decadiene with DDSQ-2SiH proceeds with lower selectivity as formation of $\alpha$-hydrosilylation product is observed resonanse lines at $(-19.05 \mathrm{ppm})$. The ${ }^{29} \mathrm{Si}$ NMR of DDSQ-2Si-Decenyl obtained via condensation and hydrosilylation procedure is presented on Fig. 4.

All obtained products are air-stable solids or waxy solids and can be synthesised on a multigram scale. They are soluble in organic solvents like DCM, $\mathrm{CHCl}_{3}$, THF and toluene but not in, e.g. methanol, $\mathrm{MeCN}$ and hexane. They were isolated an characterized by means of spectroscopic $\left({ }^{1} \mathrm{H},{ }^{13} \mathrm{C}\right.$ and ${ }^{29} \mathrm{Si}$ NMR as well as FT-IR, see Supporting Information) methods.

\section{Conclusions}

We prepared novel dialkenyl-functional double-decker silsesquioxanes with various chain lengths, from allyl, hex-5-enyl- to dec-9-enyl. We revealed simple and efficient ways of their synthesis and isolation with high 


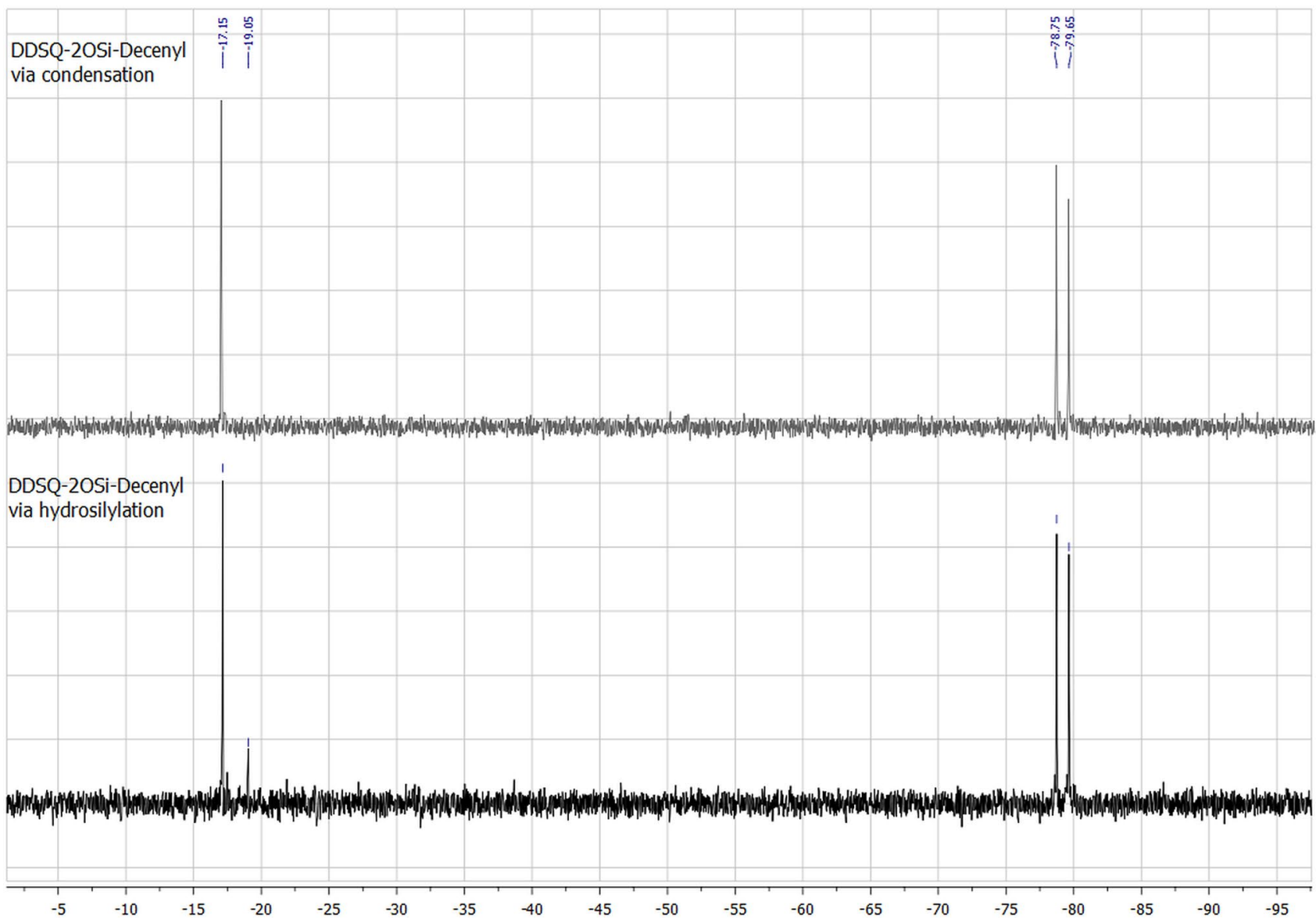

Fig. $4{ }^{29} \mathrm{Si}$ NMR spectra of DDSQ-2Si-Decenyl via condensation and hydrosilylation reaction

purity using either condensation reaction with respective dichlorosilanes or hydrosilylation of 1,5-hexadiene or 1,9-decadiene with double-decker silsesquioxane with two reactive $\mathrm{Si}-\mathrm{H}$ moieties. Both processes proved to be an alternative in the case of longer alkenyl chain length. For allyl derivative, the condensation was the only mean of obtaining this compound. The study presented is an important element in the search for new silsesquioxane based precursors and efficient methods of their syntheses. The possible application of these dialkenylfunctional DDSQ based systems is wide, e.g. they can be used as comonomers in synthesis of copolymers with ethylene [15].

\section{Experimental Section}

\subsection{Materials and Methods}

The chemicals were purchased from the following sources: dichloromethylsilane, 1,5-hexadien, Karstedt's catalyst (platinum(0)-1,3-divinyl-1,1,3,3-tetramethyldisiloxane complex $[\mathrm{Pt}(\mathrm{dvs})]$, solution in xylene with $2 \%$ of $\mathrm{Pt})$, chloroform-d, calcium hydride, lithium aluminium hydride (LAH), molecular sieves type $4 \AA$, triethylamine, $N, N$-diisopropylethylamine from Sigma-Aldrich. Allyldichloromethylsilane, 1,9-decadiene from TCI. DDSQ tetrasilanol form $\left(\mathrm{C}_{48} \mathrm{H}_{44} \mathrm{O}_{14} \mathrm{Si}_{8}\right)$ (DDSQ$4 \mathrm{OH}$ ) from Hybrid Plastic. Toluene, tetrahydrofuran (THF), methanol, dichloromethane (DCM) from P.O.Ch Gliwice. Silica gel-MN-Kieselgel 60 from Fluka Chemie AG. TLCsheets from Macherey-Nagel. Sartorius Helium from Air Products. Argon and liquid nitrogen from Linde Gas. All syntheses were conducted under argon atmosphere using standard 
Schlenk-line and vacuum techniques. Toluene was dried over $\mathrm{CaH}_{2}$ and THF over $\mathrm{LiAlH}_{4}$ (LAH) prior to use and stored under argon over type $4 \mathrm{~A}$ molecular sieves. Amines were dried over $\mathrm{CaH}_{2}$ prior to use and stored under argon in Schlenk.

\subsection{Measurements}

${ }^{1} \mathrm{H}$ NMR, ${ }^{13} \mathrm{C}$ and ${ }^{29} \mathrm{Si}$ NMR spectra measurements were conducted using a spectrometer-Bruker Ultrashield 300 and $400 \mathrm{MHz}$, using $\mathrm{CDCl}_{3}$ as a solvent. Chemical shifts are reported in $\mathrm{ppm}$ with reference to the residual solvent $\left(\mathrm{CHCl}_{3}\right)$ peaks for ${ }^{1} \mathrm{H}$ and ${ }^{13} \mathrm{C}$ and to TMS for ${ }^{29} \mathrm{Si}$. Fourier Transform-Infrared (FT-IR) spectra were recorded on a Bruker Tensor 27 Fourier transform spectrophotometer equipped with a SPECAC Golden Gate, diamond ATR unit with a resolution of $2 \mathrm{~cm}^{-1}$. GC Analyses were performed on Bruker 430 gas chromatograph equipped with a TCD detector and capillary column megabore HP-130 m (Hewlett Packard). Thin Layer Chromatography (TLC) was conducted on TLC-sheets coated with $0.2 \mathrm{~mm}$ thick silica gel with fluorescent indicator $\mathrm{UV}_{254}$. Column chromatography was performed on silica gel 60 (70-230 mesh) using hexane/DCM.

\subsection{Synthetic Procedures}

\subsubsection{Preparation of DDSQ-2Si-H $-\mathrm{Ph}_{8}\left(\mathrm{Si}_{10} \mathrm{O}_{14}\right) \mathrm{H}_{2} \mathrm{Me}_{2}-\mathrm{Di}[9$, 13-Hydromethyl]-1,3,5,7,11,13,15,17-Octa(Phenyl) Pentacyclo-[11.7.1.1 $1^{3,11} \cdot 1^{5,17} \cdot 1^{7,15}$ ]Decasiloxane [11]}

DDSQ-4OH (2.08 g, $1.95 \mathrm{mmol})$, anhydrous THF (50 mL) and (iPr) ${ }_{2} \mathrm{NEt}(1.53 \mathrm{~mL}, 8.96 \mathrm{mmol})$ were added into a twoneck round-bottom flask equipped with a magnetic stirrer. The reaction mixture was placed in ice-water bath and purged with highly pure argon. Then dichloromethylsilane $(0.44 \mathrm{~mL}$, $4.09 \mathrm{mmol}$ ) was added. Reaction was carried out for $24 \mathrm{~h}$ at room temperature. Then insoluble solid of $\left[\mathrm{HN}(\mathrm{iPr})_{2} \mathrm{Et}\right] \mathrm{Cl}$ was removed by filtration on a glass frit, volatiles and THF were eliminated via rotary evaporation. The crude product was transferred onto chromatographic column (silica gel 60) using DCM : hexane 3:2 as an eluent. Evaporation of eluent gave an analytically pure sample with $94 \%$ yield $(2.1 \mathrm{~g})$.

${ }^{1} \mathbf{H}$ NMR $\left(\mathrm{CDCl}_{3}\right) \delta(\mathrm{ppm}): 0.37$ (s, 6H, $\left.\mathrm{SiMe}_{2}\right), 4.98$ (s, 1H, Si-H), 7.16-7.57 (m, 40H, Ph). ${ }^{13} \mathbf{C}$ NMR $\left(\mathrm{CDCl}_{3}\right) \delta$ (ppm): 0.63 (-SiMe), 127.65-127.83, 130.50, 133.93-134.10 $(-\mathrm{Ph}) .{ }^{29} \mathrm{Si} \mathrm{NMR}\left(\mathrm{CDCl}_{3}\right) \delta(\mathrm{ppm}):-32.80,-77.84,-79.09$, $-77.29,-79.49$.

\subsection{Synthetic Procedure for Preparation of Dichloro(Alkenyl)Methylsilane}

\subsubsection{Preparation of Dichloro(Dec-9-Enyl)Methylsilane Via Hydrosilylation Reaction}

1,9-Decadiene $(38.6 \mathrm{ml}, 198.95 \mathrm{mmol})$ and $\left[\mathrm{Pt}_{2}(\mathrm{dvds})_{3}\right]$ $\left(0.11 \mathrm{~mL}, 9.95 \times 10^{-6} \mathrm{~mol}\right)$ were added into a two-neck round-bottom flask equipped with a magnetic stirrer and condenser. The reaction mixture was purged with highly pure argon. Then dichloromethylsilane $(5 \mathrm{~mL}, 49.70 \mathrm{mmol})$ was added. Reaction was carried out for $24 \mathrm{~h}$ at room temperature. The reaction mixture was distilled under reduced pressure $(0.17$ bar $)$ and the excess of 1,9-decadiene was removed, and final product was distilled at $65{ }^{\circ} \mathrm{C}$ in good yield $91 \%$. There was up to $5 \%$ of unreacted 1,9 -decadiene present in the obtained dichloro(dec-9-enyl)methylsilane (due to distillation under reduced pressure and resulting lowering boiling points temperatures). Due to corrosive nature of chlorosilane this mixture was used in the condensation reaction since, it should be emphasized, 1,9-decadiene is inert these conditions.

${ }^{1} \mathbf{H}$ NMR $\left(\mathrm{CDCl}_{3}\right) \delta(\mathrm{ppm}): 0.77\left(\mathrm{~s}, 3 \mathrm{H}, \mathrm{CH}_{3} \mathrm{Si}-\right)$, $1.08-1.14\left(\mathrm{~m}, 2 \mathrm{H},-\mathrm{CH}_{2}-\right), 1.29-1.38\left(\mathrm{~m}, 12 \mathrm{H},-\mathrm{CH}_{2}-\right)$, 2.01-2.08 (m, 2H, $\left.-\mathrm{CH}_{2}-\right), 4.91-5.03\left(\mathrm{~m}, 2 \mathrm{H}, \mathrm{CH}_{2}=\mathrm{CH}-\right)$, 5.75-5.88 (m, $\left.1 \mathrm{H}, \mathrm{CH}_{2}=\mathrm{CH}-\right)$.

${ }^{13} \mathbf{C ~ N M R}\left(\mathrm{CDCl}_{3}\right) \delta(\mathrm{ppm}): 5.35\left(\mathrm{CH}_{3} \mathrm{Si}-\right), 21.76$ $\left(-\mathrm{CH}_{2}-\right), 22.54\left(-\mathrm{CH}_{2}-\right), 29.03\left(-\mathrm{CH}_{2}-\right), 29.21\left(-\mathrm{CH}_{2}-\right)$, $29.23\left(-\mathrm{CH}_{2}-\right), 29.40\left(-\mathrm{CH}_{2}-\right), 32.48\left(-\mathrm{CH}_{2}-\right), 33.94$ $\left(-\mathrm{CH}_{2}-\right), 114.32\left(\mathrm{CH}_{2}=\mathrm{CH}-\right), 139.32\left(\mathrm{CH}_{2}=\mathbf{C H}-\right)$.

${ }^{29} \mathrm{Si} \mathrm{NMR}\left(\mathrm{CDCl}_{3}\right) \delta(\mathrm{ppm}): 32.95$.

MS (75 eV) m/z (\%): 55 (57), 67 (25), 69 (55), 83 (35), 113 (100), 115 (67), 154 (76), 156 (57), 254 (1) [ $\left.\mathrm{M}^{+}\right]$.

\subsubsection{Preparation of Dichloro(Hex-5-Enyl)Methylsilane Via Hydrosilylation Reaction}

1,5-Hexadiene $(4.8 \mathrm{ml}, 39.61 \mathrm{mmol})$ and $\left[\mathrm{Pt}_{2}(\mathrm{dvds})_{3}\right]$ $\left(22.6 \times 10^{-3} \mathrm{~mL}, 1.98 \times 10^{-6} \mathrm{~mol}\right)$ were added into twoneck round-bottom flask equipped with a magnetic stirrer and condenser. The reaction mixture was purged with highly pure argon. Then dichloromethylsilane $(1 \mathrm{~mL}$, $9.90 \mathrm{mmol}$ ) was added. Reaction was carried out for $24 \mathrm{~h}$ at room temperature. The reaction mixture was distilled under atmospheric pressure and the excess of 1,5-hexadiene was removed, and final product was distilled at $88-90{ }^{\circ} \mathrm{C}$ in good yield $86 \%$. There was up to $5 \%$ of unreacted 1,5 -hexadiene present in the obtained dichloro(hex-5-enyl)methylsilane. Due to corrosive nature of chlorosilane this mixture was used in the condensation reaction since, it should be emphasized, 1,5-hexadiene is inert these conditions.

${ }^{1} \mathbf{H}$ NMR $\left(\mathrm{CDCl}_{3}\right) \delta(\mathrm{ppm}): 0.77\left(\mathrm{~s}, 3 \mathrm{H}, \mathrm{CH}_{3} \mathrm{Si}-\right)$, 1.10-1.15 (m, 2H, $\left.-\mathrm{CH}_{2}-\right), 1.46-1.57\left(\mathrm{~m}, 4 \mathrm{H},-\mathrm{CH}_{2}-\right)$, 
2.05-2.12 (m, 2H, $\left.-\mathrm{CH}_{2}-\right)$, 4.94-5.05 (m, 2H, $\left.\mathrm{CH}_{2}=\mathrm{CH}-\right)$, 5.73-5.87 (m, 1H, $\left.\mathrm{CH}_{2}=\mathrm{CH}-\right)$.

${ }^{13} \mathbf{C ~ N M R}\left(\mathrm{CDCl}_{3}\right) \delta(\mathrm{ppm}): 5.33\left(\mathrm{CH}_{3} \mathrm{Si}-\right), 21.61$ $\left(-\mathrm{CH}_{2}-\right), 22.03\left(-\mathrm{CH}_{2}-\right), 31.66\left(-\mathrm{CH}_{2}-\right), 33.38\left(-\mathrm{CH}_{2}-\right)$, $114.87\left(\mathrm{CH}_{2}=\mathrm{CH}-\right), 138.51\left(\mathrm{CH}_{2}=\mathbf{C H}-\right)$.

${ }^{29} \mathrm{Si} \mathrm{NMR}\left(\mathrm{CDCl}_{3}\right) \delta(\mathrm{ppm}): 32.76$.

MS (75 eV) $m / z(\%): 198(2)\left[\mathbf{M}^{+}\right], 156(23), 154$ (58), 117 (16), 115 (81), 113 (100), 79 (10), 70 (16), 63 (11), 57 (20), 55 (15).

\subsection{General Procedure for Preparation of DDSQ-Difunctional Compounds}

\subsubsection{Procedure for Preparation of Difunctional DDSQ Compounds Via Condensation Reaction}

4.5.1.1 An Example of Condensation Reaction to Obtain DDSQ-2Si-Decenyl The synthesis was based on the hydrolytic condensation of DDSQ tetrasilanol (DDSQ-4OH) with the respective dichloroalkenylsilane carried out in the presence of triethylamine under argon atmosphere. Herein, a procedure for the synthesis of DDSQ-2Si-Decenyl $(n=8)$ is presented. DDSQ-4OH $(2.54 \mathrm{~g}, 2.38 \mathrm{mmol})$, dry THF $(35 \mathrm{~mL})$ and triethylamine $(1.99 \mathrm{~mL}, 14.25 \mathrm{mmol})$ were added into two-neck round-bottom flask equipped with a magnetic stirrer and placed in ice bath. Then dichloro(dec9-enyl)methylsilane (1.91 mL, $4.99 \mathrm{mmol})$ was introduced by dropping to the obtained solution. The reaction mixture became cloudy as a result of hydrochloride formation and consequently $\left[\mathrm{HNEt}_{3}\right] \mathrm{Cl}$ was observed as a white solid. After $1 \mathrm{~h}$, the mixture was warmed to room temperature and continuously stirred for $23 \mathrm{~h}$. Then the post-reaction mixture was subjected to filtration in order to remove the formed triethylamine hydrochloride. The filtrate was evaporated under reduced pressure. The crude product was transferred onto chromatographic column (silica gel 60) using DCM:hexane 3:2 as an eluent. Evaporation of eluent gave an analytically pure sample with $94 \%$ yield.

The same procedure and stoichiometry were applied to obtain DDSQ-2-Si-Allyl $(\mathrm{n}=1)$. The crude product, after filtration from $\left[\mathrm{HNEt}_{3}\right] \mathrm{Cl}$ and evaporation of THF, was dissolved in DCM and precipitated in $\mathrm{MeOH}$. After evaporation of $\mathrm{MeOH}$, DDSQ-2Si-All was isolated as a white solid, with $70 \%$ yield.

The same procedure and stoichiometry were applied to obtain DDSQ-2-Si-Hexenyl $(n=4)$. The crude product, after filtration from $\left[\mathrm{HNEt}_{3}\right] \mathrm{Cl}$ and evaporation of THF, was dissolved in DCM and precipitated in $\mathrm{MeOH}$. After evaporation of MeOH, DDSQ-2-Si-Hexenyl was isolated as white solid, with $68 \%$ yield.

\subsubsection{Procedure for Preparation of Difunctional DDSQ Compounds Via Hydrosilylation Reaction}

4.5.2.1 An Example of Hydrosilylation Reaction to Obtain DDSQ-2Si-Hexenyl To a $100 \mathrm{~mL}$ glass reactor equipped with a condenser and magnetic stirrer, DDSQ-2Si-H (2.00 g, $1.74 \mathrm{mmol}$ ) was placed in argon atmosphere along with toluene $(40 \mathrm{~mL}), 1,5$-hexadiene $(2.13 \mathrm{~mL}, 17.4 \mathrm{mmol})$ and $\left[\mathrm{Pt}_{2}(\mathrm{dvds})_{3}\right]\left(3.97 \mathrm{uL} 3.48 \times 10^{-7} \mathrm{~mol}\right)$. The reaction mixture was kept at $70{ }^{\circ} \mathrm{C}$ to obtain complete $\mathrm{Si}-\mathrm{H}$ consumption (usually $20 \mathrm{~h}$ ). Then, the volatiles were evaporated under vacuum and the crude product was dissolved in DCM and precipitated in $\mathrm{MeOH}$. After evaporation of $\mathrm{MeOH}$, DDSQ-2Si-Hexenyl was isolated as white solid, with $90 \%$ yield.

The same procedure and stoichiometry were applied to obtain DDSQ-2-Si-Decenyl $(\mathrm{n}=8)$. The reaction mixture was kept at $95{ }^{\circ} \mathrm{C}$ to obtain complete $\mathrm{Si}-\mathrm{H}$ consumption (usually $20 \mathrm{~h}$ ). Then toluene was evaporated under vacuum. The crude product, was washed several times with $\mathrm{MeOH}$. DDSQ-2SiDecenyl was isolated as waxy solid, with $79 \%$ yield. The solid was contaminated with traces 1,9-decadiene, occluded within the structure of a product.

Acknowledgements Financial support from the National Science Centre (Poland) Project OPUS No. DEC-2012/07/B/ST5/03042 and National Centre for Research and Development in Poland - PBS3/A1/16/2015 is acknowledged.

Open Access This article is distributed under the terms of the Creative Commons Attribution 4.0 International License (http://creativecommons.org/licenses/by/4.0/), which permits unrestricted use, distribution, and reproduction in any medium, provided you give appropriate credit to the original author(s) and the source, provide a link to the Creative Commons license, and indicate if changes were made.

\section{References}

1. D.B. Cordes, P.D. Lickiss, F. Rataboul, Chem. Rev. 110, 2081 (2010)

2. C. Hartmann-Thompson, Applications of Polyhedral Oligomeric Silsesquioxanes (Springer, New York, 2011)

3. E. Ayandele, B. Sarkar, P. Alexandridis, Nanomaterials 2, 445 (2012)

4. Q. Ye, H. Zhou, J. Xu, Chem.-Asian J. 11, 1322 (2016)

5. H. Zhou, Q. Ye, J. Xu, Mater. Chem. Front. 1, 212 (2017)

6. Y. Morimoto, K. Watanabe, N. Ootake, J. Inagaki, K. Yoshida, K. Ohguma, US 7449539 B2 (2008)

7. H. Oikawa, M. Yamahiro, K. Ohguma, N. Ootake, K. Watanabe, K. Ohno, Y. Tsujii, T. Fukuda, US 7375170 B2 (2008)

8. B. Dudziec, B. Marciniec, Curr. Org. Chem 20, 1 (2016)

9. M. Seino, T. Hayakawa, Y. Ishida, M. Kakimoto, K. Watanabe, H. Oikawa, Macromolecules 39, 3473 (2006)

10. M. Miyasaka, Y. Fujiwara, H. Kudo, T. Nishikubo, Polym. J. 42, 799 (2010)

11. M. Walczak, R. Januszewski, M. Majchrzak, M. Kubicki, B. Dudziec, B. Marciniec, New J. Chem. 41, 3290 (2017)

12. P. Zak, M. Majchrzak, G. Wilkowski, B. Dudziec, M. Dutkiewicz, B. Marciniec, RSC Adv. 6, 10054 (2016) 
13. P. Żak, B. Dudziec, M. Dutkiewicz, M. Ludwiczak, B. Marciniec, M. Nowicki, J. Polym. Sci. A 54, 1044 (2016)

14. B. Marciniec, H. Maciejewski, C. Pietraszuk, P. Pawluć, Hydrosilylation: A Comprehensive Review on Recent Advances (Springer, New York, 2009)
15. P. Groch, K. Dziubek, K. Czaja, M. Białek, B. Dudziec, B. Marciniec, Eur. Polym. J. (2017) (submitted) 